\title{
Neue Richtlinien zur Lebendspende von soliden Organen
}

Jürg Steiger ${ }^{a}$, Michelle Salathéb

a Prof. Dr. med., Vorsitzender der Subkommission

b lic. iur., stv. Generalsekretärin SAMW
* Aus Gründen der leichteren Lesbarkeit gilt in diesem Text die männliche Bezeichnung für beide Geschlechter.

Korrespondenz:

lic. iur. Michelle Salathé

SAMW

Petersplatz 13

CH-4051 Basel

Tel. 0612699030

Fax 0612699039

mail@samw.ch

www.samw.ch
Die Organtransplantation stellt heute eine bewährte und erfolgversprechende Therapieform dar. Fortschritte im Bereich der medizinischen und chirurgischen Betreuung haben zu einer Erhöhung der Lebenserwartung und einer Steigerung der Lebensqualität von Organempfängern geführt. Diese Entwicklung führt $\mathrm{zu}$ einem steigenden Bedarf an Organen. Während jedoch die Zahl von toten Spendern abnimmt, ist die Bereitschaft zur Lebendspende in der Schweiz höher. Weil die Wartelisten für Organe lang sind und Lebendtransplantationen zudem eine deutlich bessere Erfolgsaussicht haben, gewinnt die Lebendspende immer mehr an Bedeutung. Vor diesem Hintergrund hat der Senat der Schweizerischen Akademie der Medizinischen Wissenschaften (SAMW) am 20. Mai 2008 neue medizinisch-ethische Richtlinien «Lebendspende von soliden Organen» verabschiedet.

Mit dem neuen Bundesgesetz über die Transplantation von Organen und Geweben und den entsprechenden Verordnungen, die seit dem 1. Juli 2007 in Kraft sind, hat der Bund eine umfassende Regelung für diesen komplexen Bereich der Medizin geschaffen. Dies ist auch der Grund, weshalb die neuen Richtlinien diverse Querverweise auf das neue Transplantationsgesetz und dessen Ausführungsverordnungen enthalten. Die Richtlinien, die sich auf die Lebendspende von soliden Organen (namentlich Lebern und Nieren) beschränken, füllen für einmal keine gesetzliche Lücke, sondern leisten einen Beitrag zur Umsetzung der gesetzlichen Vorgaben in die Praxis. Sie richten sich an Ärzte*, Pflegende und weitere Fachpersonen, welche sich an der Abklärung potentieller Spender beteiligen.

Die gesetzliche Regelung geht vom Grundsatz aus, dass jede urteilsfähige und mündige Person nach umfassender Aufklärung eigenverantwortlich und frei entscheiden kann, ob sie das Risiko der Organspende zugunsten einer Drittperson auf sich nehmen will. Die Richtlinien weisen darauf hin, dass die involvierten Ärzte den autonomen Spenderwunsch respektieren müssen, gleichzeitig aber auch die Pflicht haben, dem Spender nicht zu schaden. Der Spenderschutz muss jedoch immer an erster Stelle stehen. Sind die Risiken für Gesundheit und Leben des Spenders zu gross, muss eine Spende abge- lehnt werden, auch wenn dies nicht dem Wunsch des Spenders entspricht. Die RisikoNutzen-Abwägung im Einzelfall hängt von vielen Faktoren ab. Beim Spenderschutz sind nicht nur medizinische Aspekte zu beachten, sondern auch psychosoziale Faktoren, wie z.B. die psychischen Folgen einer Spende bzw. NichtSpende. Eine Spende muss auch dann abgelehnt werden, wenn sich herausstellt, dass der Spendewunsch auf äusserem Druck basiert.

Die erste Fassung der Richtlinien «Lebendspende von soliden Organen» wurde in der Vernehmlassung mehrheitlich begrüsst, teilweise aber auch kritisch hinterfragt. Um den in der Vernehmlassung geäusserten Einwänden Rechnung zu tragen, hat die Subkommission unter der Leitung von Professor Jürg Steiger aus Basel die erste Fassung der Richtlinien in zentralen Punkten überarbeitet. So enthalten die Richtlinien nun zusätzliche Kapitel zur Abklärung der Freiwilligkeit oder zum Umgang mit Spendern, die Bluttransfusionen ablehnen, und die für die Lebendspende relevanten ethischen Überlegungen werden ausführlicher dargelegt. Die Richtlinien sehen neu auch vor, dass bei Zweifeln über die Spendereignung - unter Wahrung der Persönlichkeitsrechte des Spender-EmpfängerPaares - eine externe Meinung eingeholt werden kann.

Mit den Richtlinien «Lebendspende von soliden Organen» will die SAMW Ärztinnen und Ärzte, Pflegende und weitere Fachpersonen bei der medizinischen und psychosozialen Abklärung der Spender und deren Nachbetreuung unterstützen. Wie die meisten Richtlinien der SAMW enthalten auch diese zahlreiche Empfehlungen an die Transplantationszentren, die Gesundheitsdirektorenkonferenz, den Gesetzgeber und die Versicherer, welche dazu beitragen sollen, dass die Richtlinien in der Praxis umgesetzt werden können.

Die Richtlinien können auf der Website der SAMW (www.samw.ch $\rightarrow$ ETHIK) heruntergeladen oder beim Generalsekretariat der SAMW, Petersplatz 13, 4051 Basel, gratis als Broschüre bestellt werden. 